\title{
A New Charged Anisotropic Compact Star Model in General Relativity
}

\author{
Ritu Tamta, Pratibha Fuloria \\ Physics Department, S.S.J. Campus, Almora, India \\ Email: ritutamta87@gmail.com,p.fuloria@yahoo.com
}

How to cite this paper: Tamta, R. and Fuloria, P. (2017) A New Charged Anisotropic Compact Star Model in General Relativity. Journal of Modern Physics, 8, 1762-1778.

https://doi.org/10.4236/jmp.2017.811104

Received: August 26, 2017

Accepted: October 8, 2017

Published: October 11, 2017

Copyright (c) 2017 by authors and Scientific Research Publishing Inc. This work is licensed under the Creative Commons Attribution International License (CC BY 4.0).

http://creativecommons.org/licenses/by/4.0/

\begin{abstract}
In the present article, we explore a new static, spherically symmetric charged anisotropic fluid model of compact star in curvature coordinates. We consider metric potential $g_{44}$ of Durgapal's fifth solution [1] with a specific choice of electric field intensity $E$ and physically acceptable expression of anisotropy factor $\Delta$, which involve parameters $K$ (charge) and $\Delta$ (anisotropy) respectively. The solution so obtained is utilized to construct the model for super-dense star like neutron star. We have analysed that corresponding to $X=0.1, \quad K=2.8, \quad \alpha=1.6$ and by assuming surface density $\rho_{b}=2 \times 10^{14} \mathrm{gm} / \mathrm{cm}^{3}$, the mass of the compact star comes out to be $2.17 M_{\Theta}$ with radius $14.51 \mathrm{kms}$, which closely resembles to that of PSRJ0348 + 0432 . The solution is well behaved for the values of $K$ satisfying $1 \leq K<5$. Our model is described analytically as well as with the help of graphical representations. Our solution is well behaved and free from any central singularity. It also satisfies all the energy conditions as well as the causality condition thus reconfirming the stability of our model.
\end{abstract}

\section{Keywords}

General Relativity, Neutron Star, Anisotropy, Exact Solutions

\section{Introduction}

During the final stages of stellar evolution when the nuclear fuel inside the star is almost exhausted, the star deviates from its hydrostatic equilibrium stage and starts to collapse. During contraction, gravitational force dominates all other existing forces i.e. pressure gradient forces and radiation forces. The collapse will continue until some quantum-mechanical phenomena such as electron degeneracy pressure, neutron degeneracy pressure generates inside the star thereby 
providing stability to it against gravitational collapse. Compact objects such as white dwarf stars, neutron stars, strange stars and black holes represent the final stages of a star's evolution. The stars at this stage become so dense that relativistic effects come into picture and have significant role in describing the crucial features of compact stars. The possibility of existence of Quark star and strange star at such high densities can not be ignored. Neutron star is composed of neutrons, while strange star is composed of $u, d$, and s quarks. The formation of strange matter can be categorized in two ways: the quark hadron phase transition in the early universe and conversion of neutron stars into strange stars [2]. When a massive star undergoes a supernova explosion, neutron star and strange quark star are expected to be formed at the inner core of the star. A phase transition between hadronic and strange quark matter may occur at such a higher densities [3]. Recent developments in observation cosmology have explored interesting facts regarding evolution of these compact objects and revealed many of their features from the study of their emission spectra. But still some of the parameters like mass, internal composition, radii, etc. are not clearly known which cannot be inferred from direct observational data. However many theoretical investigations have successfully explained the various characteristics of compact stellar objects. The study of relativistic models of compact stars like neutron stars and strange stars have been a field of active research in recent years. To understand the nature and complex composition of such immense gravity objects, we require an exact solution to the Einstein's field equations. The first exact solution of the Einstein's field equations was obtained by K Schwarzschild in 1916 describing the exterior of a spherically symmetric matter distribution [4]. Later on the interior solution for a uniform density sphere was also obtained by Schwarzshild [5]. Of course no astrophysical configuration has a perfect fluid distribution. Observations of stars and the understanding of Particle Physics within compact stars have emphasized the search for more realistic solutions of the field equations. It is interesting to note that by relaxing the condition of a perfect fluid and allowing for pressure anisotropy and charge within the interior of stellar configurations, more realistic structure of compact stellar objects can be realized. It has been suggested that in the presence of charge the gravitational collapse of a spherically symmetric astrophysical object to a point singularity may be averted. The presence of charge in a charged fluid has tendency to oppose the gravitational collapse. This property persuaded the research workers to work on the charged perfect fluid configurations.

Ivanov [6] and Bonner [7] investigated a model for charged perfect fluid sphere in which formation of singularity is avoided. Many static fluid spheres which do not satisfy some or all the physical requirements become relevant after the inclusion of charge in them [8] [9]. The central density of most of compact objects could be several times higher than the normal nuclear matter density. The theoretical investigations [10] [11] [12] [13] [14] on compact stellar models strongly suggest that the matter distribution in such massive stellar objects may 
be locally anisotropic, the radial pressure may not be equal to the tangential pressure, at least in certain very high density ranges $\left(>10^{15} \mathrm{gm} \cdot \mathrm{cm}^{-3}\right)$. During the post main-sequence of a star, the surface magnetic field reaches up to $10^{12}$ $10^{13} \mathrm{G}$ when neutron star forms. Due to very intense magnetic field of a newly born NS, the matter inside the star may generate pressure anisotropy [15]. The observational evidence for the neutron star is obtained from the pulsars and its glitches. The sudden change in the time period of pulsars, called glitches is due to neutron superfluidity. Neutron superfluidity also affects the neutrinos emission rate through modified Urca process [16]. At a density of the order of $10^{15}$ $\mathrm{gm} \cdot \mathrm{cm}^{-3}$ nuclear matter may be anisotropic and it needs to be treated relativistically [17]. Poin condensation at nuclear densities may also cause anisotropy [18]. Sokolov [19] suggested that phase transition from normal states of pions to superconducting states can generate pressure anisotropy. Thus it is desirable to study the implications of Einstein-Maxwell field equations with the incorporation of anisotropy for the proper understanding of compact stars. Dev and Gleiser [20] have shown that pressure anisotropy affects the physical properties and structure of stellar matter and also provides more stability to stellar objects against radial adiabatic perturbations as compared to isotropic matter. Bowers and Liang [21] have studied anisotropic spheres with uniform energy density and suggested that anisotropy could also play an important role in describing the high redshift objects like quasars. Anisotropy in pressure could significantly affect the physical parameters like maximum compactness, mass and radius of star. A large number of anisotropic models are available in the literature [22]-[35]. Local anisotropy in self-gravitating systems were studied by Herrera \& Santos [36] They conjectured that an anisotropic model can be stable. Charged anisotropic models of stellar objects have been discussed by many Astrophysicists i.e. Thirukkanesh and Ragel [37], Maurya and Gupta [38], Pandya et al [39], Bhar et al. [40] and Murad [41]. Compact stellar models for neutral/charged static spherically symmetric configuration with anisotropic pressure have been obtained in numerous works. Some of them include; Herrera et al. [42] [43], Chaisi and Maharaj [44], Maurya and Gupta [45] [46], Maharaj et al. [47]. Pant et al. [48] have also studied a class of relativistic anisotropic charged stellar models in isotropic coordinates. There have been several recent investigations of static fluid configuration incorporating charge and pressure anisotropy: Pradhan \& Pant [49], Newton Singh et al. [50], Pant et al. [51] and Maharaj et al. [52]. Being motivated by the aforementioned recent works we develop some new analytical relativistic anisotropic charged stellar models by using Durgapal's metric potential [1]. Our analysis depends on several mathematical key assumptions. The form of metric potential ensures that the metric function is nonsingular, continuous, and well behaved in the interior of the star. This is one of the desirable features for the model on physical grounds. Further, we assume some particular forms of electric charge distribution function and pressure anisotropy so that we may construct a physically reasonable models of stellar objects.

Our whole investigation is divided into 7 sections. In first we give a brief in- 
troduction on which our present study is based on. The next section contains the complete procedure to solve Einstein Maxwell's field equations for anisotropic fluid charge distribution. In a third section we give the elementary criteria to be satisfied by the interior solution in order to present a realistic model. In the last section we conclude all results obtained after complete analysis of newly obtained solution.

\section{Einstein Maxwell Field Equations of Anisotropic Charged Fluid Distribution}

Let us consider a spherical symmetric metric in curvature coordinates as

$$
\mathrm{d} s^{2}=-e^{\lambda} \mathrm{d} r^{2}-r^{2} \mathrm{~d} \theta^{2}-r^{2} \sin ^{2} \theta \mathrm{d} \Phi^{2}+e^{v} \mathrm{~d} t^{2}
$$

For a charged and anisotropic fluid distribution the Einstein Maxwell field equation is given as

$$
-\frac{8 \pi G}{c^{4}} T_{j}^{i}=R_{j}^{i}-\frac{1}{2} R \delta_{j}^{i}
$$

Here $T_{j}^{i}$ is the energy momentum tensor and is given by

$$
T_{j}^{i}=\left[\left(p_{\perp}+\rho c^{2}\right) v^{i} v_{j}-p_{\perp} \delta_{j}^{i}+\left(p_{r}-p_{\perp}\right) \chi_{j} \chi^{i}+\frac{1}{4 \pi}\left(-F^{i m} F_{j m}+\delta_{j}^{i} F_{m n} F^{m n}\right)\right]
$$

Here $R_{j}^{i}$ is Ricci tensor, $T_{j}^{i}$ is the energy momentum tensor, $R$ is the scalar curvature, $F^{m n}$ is electromagnetic field tensor, $\left(p_{r}, p_{\perp}\right)$ are the radial and transverse pressures respectively, $\rho$ is the energy density, $v^{i}$ is the velocity vector and $\chi^{i}$ is the unit space vector in radial direction.

In view of metric (2.1), the field Equation (2.2) gives

$$
\begin{gathered}
\frac{8 \pi G}{c^{4}} p_{r}=\frac{v^{\prime}}{r} e^{-\lambda}-\frac{1-e^{-\lambda}}{r^{2}}+\frac{q^{2}}{r^{4}} \\
\frac{8 \pi G}{c^{4}} p_{\perp}=\mathrm{e}^{-\lambda}\left(\frac{v^{\prime \prime}}{2}-\frac{\lambda^{\prime} v^{\prime}}{4}+\frac{v^{\prime 2}}{4}+\frac{v^{\prime}-\lambda^{\prime}}{2 r}\right)-\frac{q^{2}}{r^{4}} \\
\frac{8 \pi G}{c^{2}} \rho=\frac{\lambda^{\prime}}{r} \mathrm{e}^{-\lambda}+\frac{1-\mathrm{e}^{-\lambda}}{r^{2}}-\frac{q^{2}}{r^{4}} \\
\sigma(r)=\frac{e^{-\lambda / 2}}{4 \pi r^{2}}\left(r^{2} E\right)^{\prime}
\end{gathered}
$$

Here $\sigma$ is the proper charge density and $\mathrm{q}$ is the charge enclosed by a sphere of radius $r$.

Assuming

$$
e^{-\lambda}=Y, e^{v}=B(1+x)^{5} \text { and } x=c_{1} r^{2}
$$

Here $B$ is the constant which has positive value. In view of Equation (2.8), Equation (2.4) and Equation (2.5) reduces to

$$
\frac{\mathrm{d} Y}{\mathrm{~d} x}+\frac{14 x^{2}-2 x-1}{x(1+x)(1+6 x)} Y+\frac{1+x}{1+6 x}\left\{\frac{1}{x}-\frac{\Delta}{c_{1}}-\frac{2 E^{2}}{c_{1}}\right\}=0
$$


Here $\Delta=\frac{8 \pi G}{c^{4}}\left(p_{\perp}-p_{r}\right)$ is anisotropy factor.

\section{Physical Acceptability Conditions}

For well behaved nature of the solution in curvature coordinates, the following conditions should be satisfied:

1) The solution should be free from physical and geometric singularities, i.e. it should yield finite and positive values of the central pressure, central density and nonzero positive value of $e^{\lambda}$ and $e^{v}$.

2) The solution should have positive and monotonically decreasing expressions for pressure and density ( $p$ and $\rho$ ) with the increase of $r$. The ratio of pressure and density should be positive and less than 1 (weak energy condition) and less than $1 / 3$ (strong energy condition) throughout within the star.

3) The casualty condition should be obeyed i.e. velocity of sound should be less than that of light throughout the model. In addition to the above the velocity of sound should be decreasing towards the surface i.e. $\frac{\mathrm{d}}{\mathrm{d} r}\left(\frac{\mathrm{d} p_{r}}{\mathrm{~d} \rho}\right)<0$ $\left(\frac{\mathrm{d}^{2} p_{r}}{\mathrm{~d} \rho}\right)>0$ and $\frac{\mathrm{d}}{\mathrm{d} r}\left(\frac{\mathrm{d} p_{t}}{\mathrm{~d} \rho}\right)<0 \quad\left(\frac{\mathrm{d}^{2} p_{t}}{\mathrm{~d} \rho}\right)>0$ or i.e. the velocity of sound is increasing with the increase of density and it should be decreasing outwards.

4) The adiabadtic index $\gamma=\frac{\rho+p_{r}}{p_{r}} \frac{\mathrm{d} p_{r}}{\mathrm{~d} \rho}$ for realistic matter should be $\gamma \geq 4 / 3$.

5) The red shift $z$ should be positive, finite and monotonically decreasing in nature with the increase of $r$.

6) Electric field intensity $E$ is positive and monotonically increasing from center to boundary and at the centre the electric intensity is zero.

7) The anisotropy factor $\Delta$ should be zero at the center and increasing towards the surface.

8) For a stable anisotropic compact star, $0<\left|v_{t}^{2}-v_{r}^{2}\right| \leq 1$ must be satisfied [24].

\section{A New Class of Solution}

To solve Equation (2.9) we assume

$$
\frac{\Delta}{c_{1}}=\frac{\alpha x(1+6 x)^{-1 / 3}}{(1+x)^{4}} \text { and } \frac{E^{2}}{c_{1}}=\frac{c_{1} q^{2}}{x^{2}}=\frac{k x(1+6 x)^{-m / 3}}{(1+x)^{4}}
$$

where $\alpha, k, l$ and $m$ are real and positive constants. We assume anisotropy and electric field intensity in such a way that Equation (2.9) is integrable and provides well behaved solutions. The solutions are physically acceptable when both anisotropy and electric field intensity increase as we move from the centre to the surface. On plugging Equation (4.1) into Equation (2.9) we get the following solution, 


$$
\begin{aligned}
e^{-\lambda}= & Y=\frac{1}{(1+x)^{3}}\left[\frac{112-309 x-54 x^{2}-8 x^{3}}{112}+\frac{\alpha x(1+6 x)^{-l / 3}}{2(1-l)}\right. \\
& \left.+\frac{k x(1+6 x)^{-m / 3}}{1-m}\right]+\frac{A x(1+6 x)^{-1 / 3}}{(1+x)^{3}}
\end{aligned}
$$

A is an arbitrary constant of integration.

The expressions for density and pressure are given by

$$
\begin{aligned}
\frac{8 \pi G}{c_{1} c^{4}} p_{r}= & \frac{475-4125 x-1050 x^{2}-200 x^{3}}{112(1+x)^{4}}+\frac{\alpha(11 x+1)(1+6 x)^{-l / 3}}{2(1-l)(1+x)^{4}} \\
& +\frac{k(12 x-m x+1)(1+6 x)^{-m / 3}}{(1-m)(1+x)^{4}}+\frac{A(11 x+1)(1+6 x)^{-1 / 3}}{(1+x)^{4}} \\
\frac{8 \pi G}{c_{1} c^{2}} \rho= & \frac{1935+15 x+450 x^{2}+120 x^{3}}{112(1+x)^{4}} \\
& -\frac{\alpha(1+6 x)^{l / 3-1}}{2(1-l)(1+x)^{4}}\left(3+15 x-4 l x-18 x^{2}-4 l x^{2}\right) \\
& -\frac{k(1+6 x)^{-m / 3-1}}{(1-m)(1+x)^{4}}\left(3+15 x-4 m x-18 x^{2}-4 m x^{2}\right) \\
& -\frac{k x(1+6 x)^{-m / 3}}{(1+x)^{4}}-\frac{A(1+6 x)^{-4 / 3}}{(1+x)^{4}}\left(-22 x^{2}+11 x+3\right)
\end{aligned}
$$

The proper charge density will be given by the following expression

$$
\sigma(x)=\frac{c_{1} \sqrt{k}}{4 \pi} Y(x) \frac{\left[3-(2 m-17) x-(6 m+2 m) x^{2}\right]}{(1+x)^{3}(1+6 x)^{1-m / 6}}
$$

Differentiating Equations (4.3), (4.4) and (4.5) with respect to r, we get the radial and tangential pressure gradients as well as density gradient.

$$
\begin{aligned}
& \frac{8 \pi G}{C_{1} c^{4}} \frac{\mathrm{d} p_{r}}{\mathrm{~d} x}= \frac{200 x^{3}+1500 x^{2}+10275 x-6025}{112(1+x)^{5}} \\
&+\frac{\alpha(1+6 x)^{-l / 3-1}}{2(1-l)(1+x)^{5}}\left(-22 l x^{2}-198 x^{2}-24 l x+9 x-2 l+7\right) \\
&+\frac{k(1+6 x)^{-m / 3-1}}{(1-m)(1+x)^{5}}\left(2 m^{2} x^{2}-6 m x^{2}-216 x^{2}\right. \\
&\left.+2 m^{2} x-29 m x+12 x-3 m+8\right) \\
&+\frac{A(1+6 x)^{-4 / 3}}{(1+x)^{5}}\left(-220 x^{2}-15 x+5\right) \\
& \frac{8 \pi G}{C_{1} C^{4}} \frac{\mathrm{d} p_{\perp}}{\mathrm{d} x}=\frac{8 \pi G}{C_{1} c^{4}} \frac{\mathrm{d} p_{r}}{\mathrm{~d} x}+\frac{\alpha(1+6 x)^{-l / 3-1}}{(1+x)^{5}}\left(-2 l x^{2}-18 x^{2}-2 l x+3 x+1\right)
\end{aligned}
$$




$$
\begin{aligned}
\frac{8 \pi G}{c_{1} c^{2}} \frac{\mathrm{d} \rho}{\mathrm{d} x}= & \frac{120 x^{3}-540 x^{2}+855 x-7725}{112(1+x)^{5}} \\
& -\frac{k(1+6 x)^{-m / 3-1}}{(1+x)^{5}}\left(-2 m x^{2}-18 x^{2}-2 m x+3 x+1\right) \\
& -\frac{A(1+6 x)^{-7 / 3}}{(1+x)^{5}}\left(440 x^{3}-330 x^{2}-195 x-25\right) \\
& -\frac{\alpha(1+6 x)^{-l / 3-2}}{2(1-l)(1+x)^{5}}\left(8 l^{2} x^{3}+108 l x^{3}+324 x^{3}+16 l^{2} x^{2}\right. \\
& \left.+86 l x^{2}-432 x^{2}+8 l^{2} x-32 l x-171 x-10 l-15\right) \\
& -\frac{k(1+6 x)^{-m / 3-2}}{(1-m)(1+x)^{5}}\left(8 m^{2} x^{3}+108 m x^{3}+324 x^{3}+16 m^{2} x^{2}\right. \\
& \left.+86 m x^{2}-432 x^{2}+8 m^{2} x-32 m x-171 x-10 m-15\right)
\end{aligned}
$$

We have shown the variation of metric potentials with radial coordinate for the star PSR J0348 + 0432 in Figure 1. It is clear from figure that metric potentials are regular and free form any singularity inside the fluid sphere. It can be observed from Figure 2 and Figure 3 that radial, transverse pressures and density monotonically decrease with the increase of radius. Electric field intensity increases when we move from centre towards surface (see Figure 4). Moreover the electric field intensity is zero at the centre, which shows that our solution satisfies the required acceptability conditions. From the graphs plotted in Figures 5-8 we notice that pressure to energy density ratio, red shift, proper charge density, square of radial and transverse velocity decrease with the increase of radial coordinate respectively. In Figure 9 we have shown the variation of anisotropy factor with radial coordinate $r$ and we observe that anisotropy factor

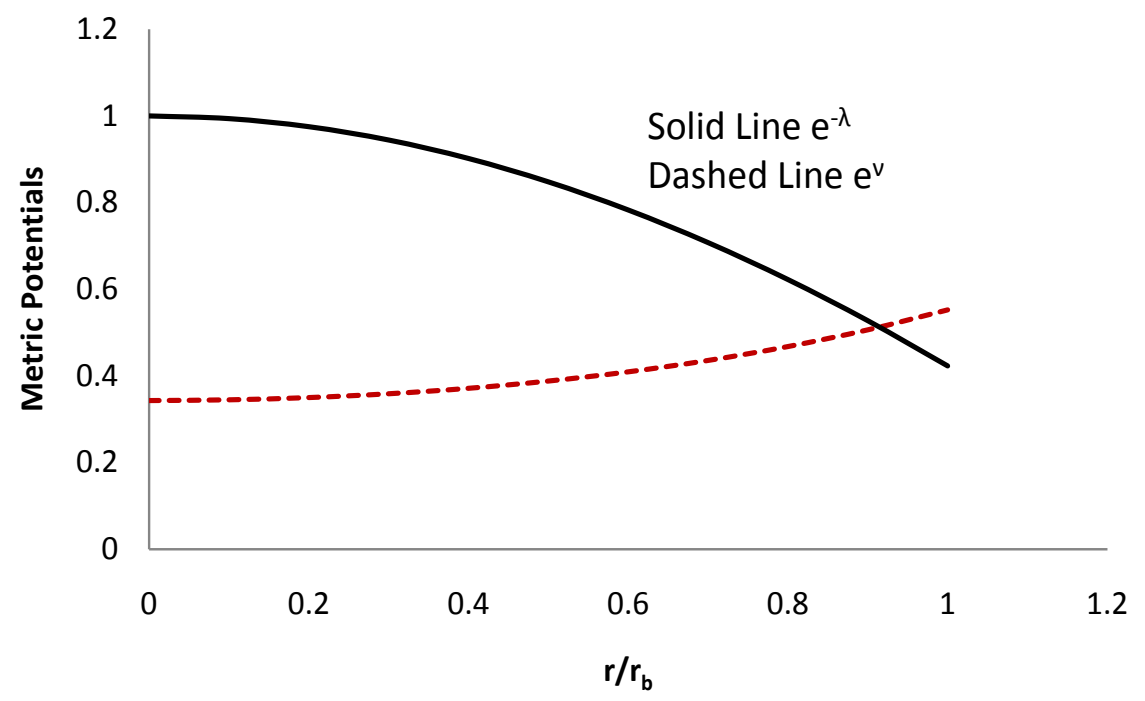

Figure 1. Variation of metric potentials with radial coordinate for PSR J0348 + 0432. 


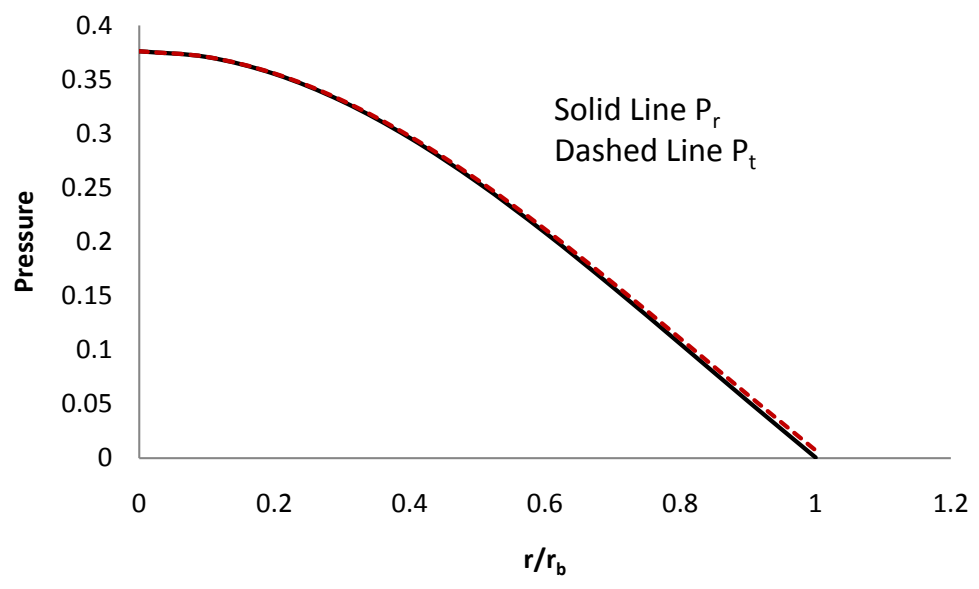

Figure 2. Variation of radial and transverse pressures with radial coordinate for PSR J0348+ 0432.

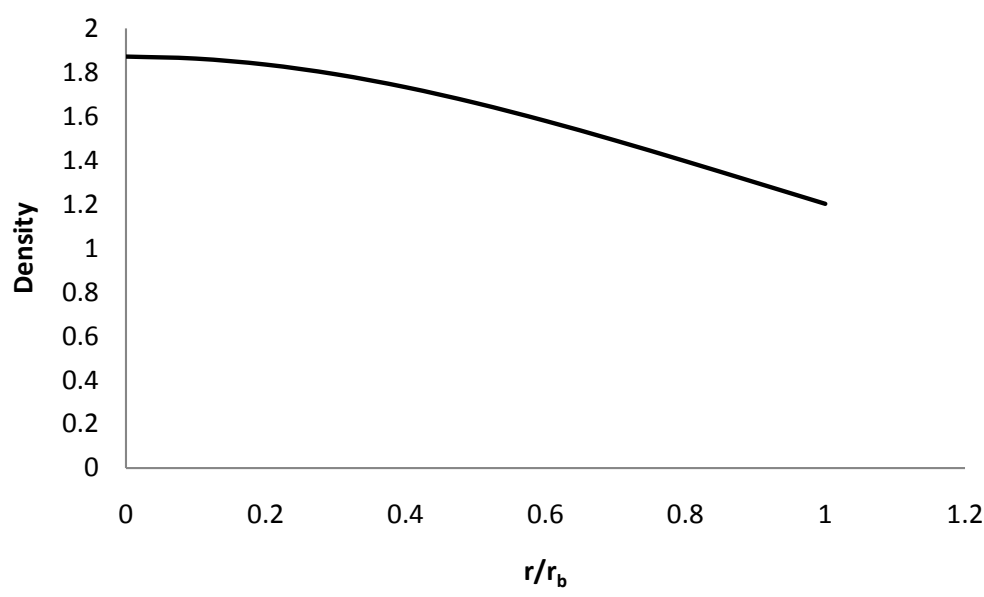

Figure 3. Variation of matter density with radius for PSR J0348 + 0432.

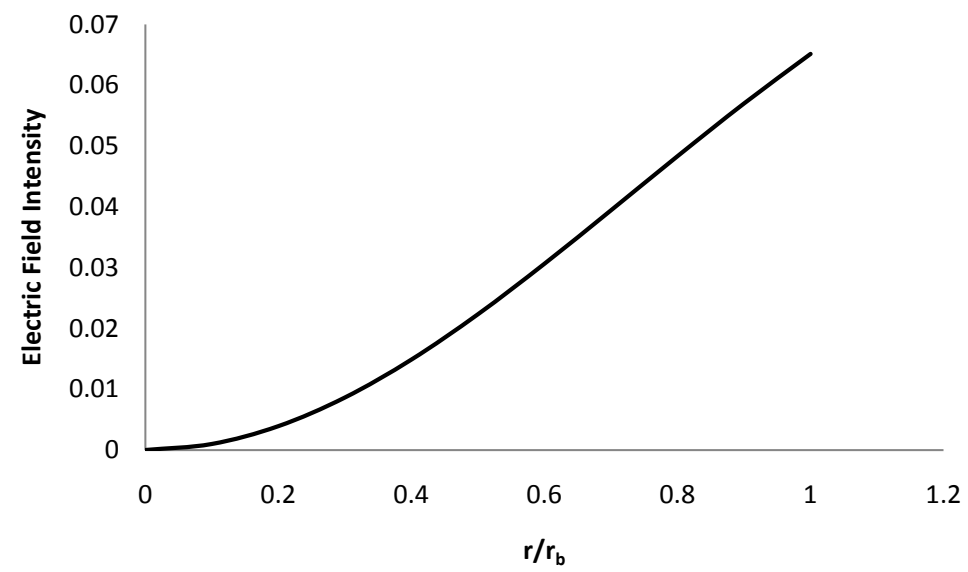

Figure 4. Variation of electric field intensity with radial coordinate for PSR $\mathrm{J} 0348+0432$.

is zero at the centre and increases in moving towards boundary. Figure 10 shows the outmarch of compression moduli with radial coordinate inside the 


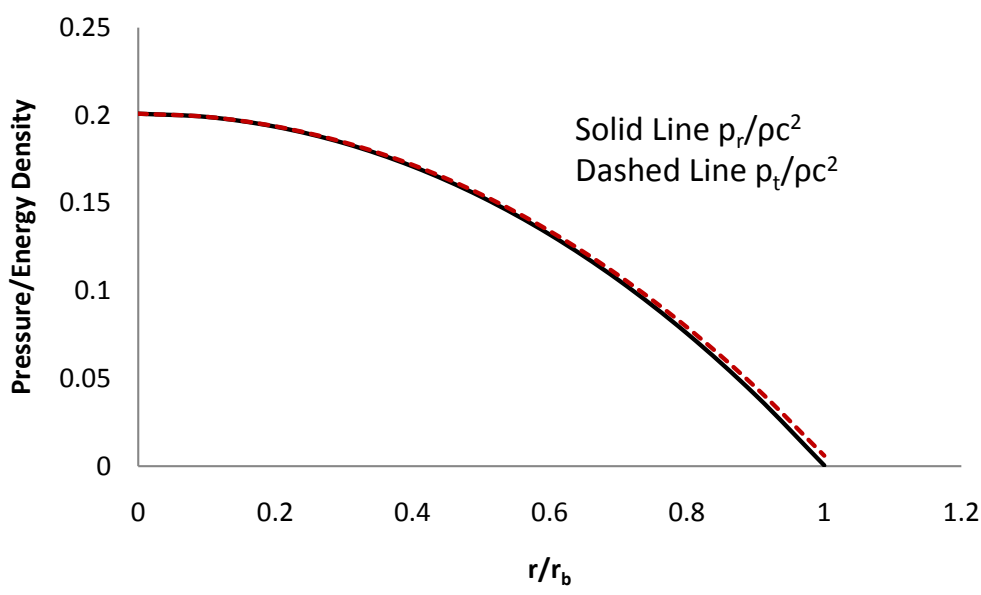

Figure 5. Variation of pressure to energy density ratio with radius for PSR $\mathrm{J} 0348+0432$.

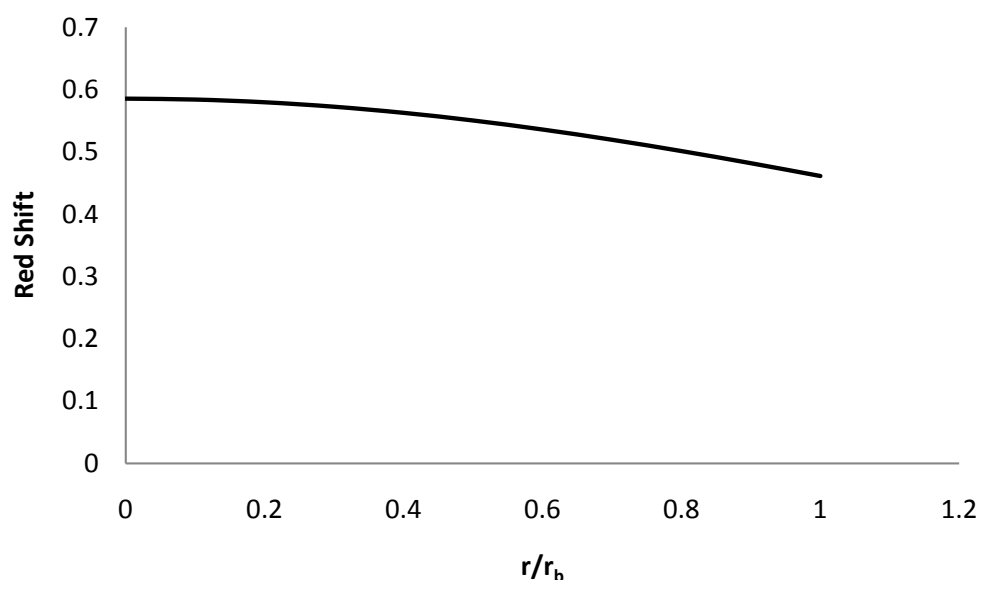

Figure 6. Variation of red shift with radial coordinate for PSR J0348+0432.

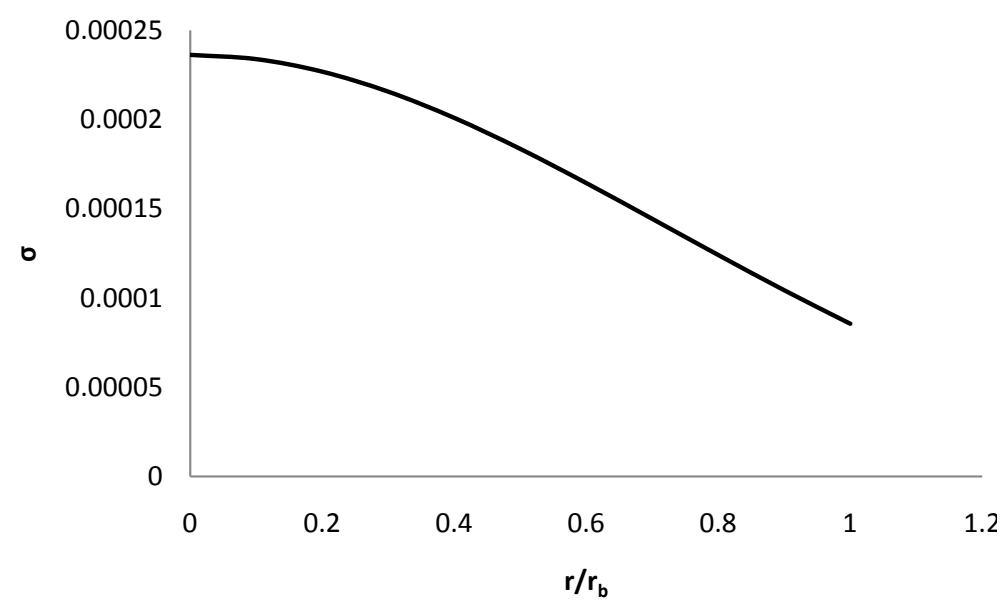

Figure 7. Variation of proper charge density with radius for PSR J0348+0432.

fluid sphere and it decreases when we move towards surface. The Null Energy Condition (NEC), Weak Energy condition (WEC) and Strong Energy Condition 


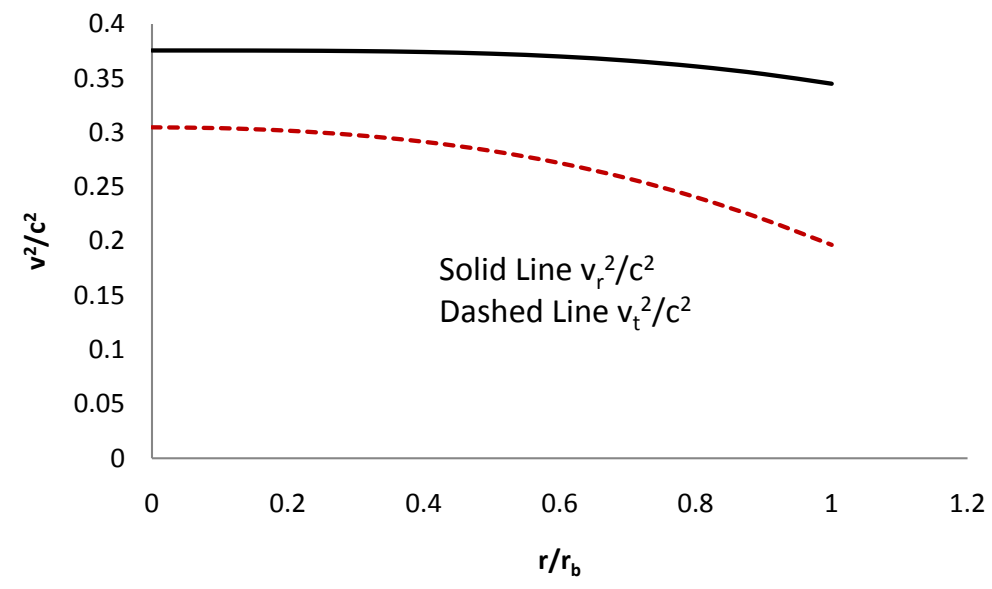

Figure 8. Variation of $\mathrm{v}^{2} / \mathrm{c}^{2}$ with radius for PSR J0348 +0432 .

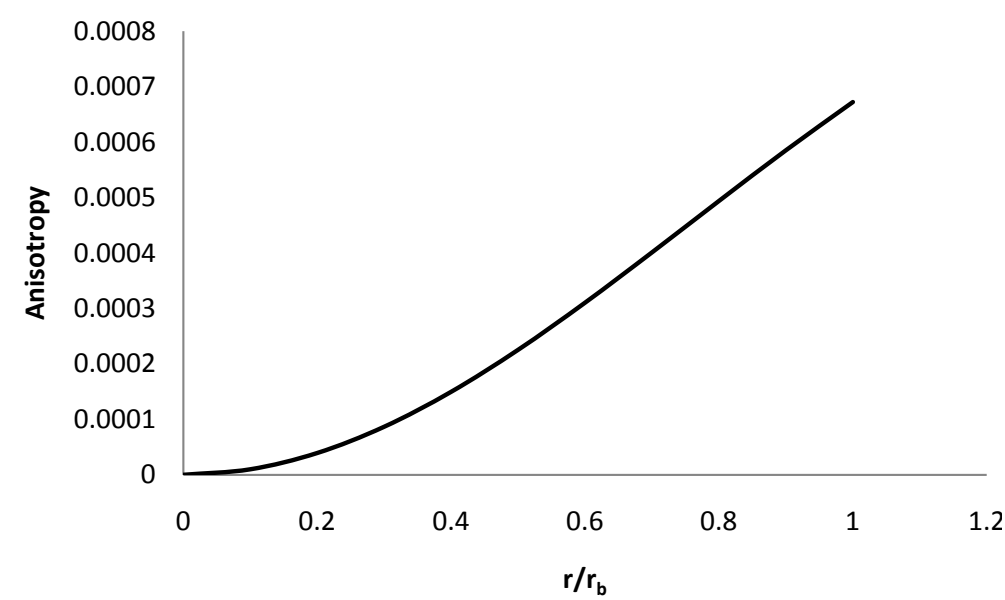

Figure 9. Variation of anisotropy with radial coordinate for PSR J0348 + 0432 .

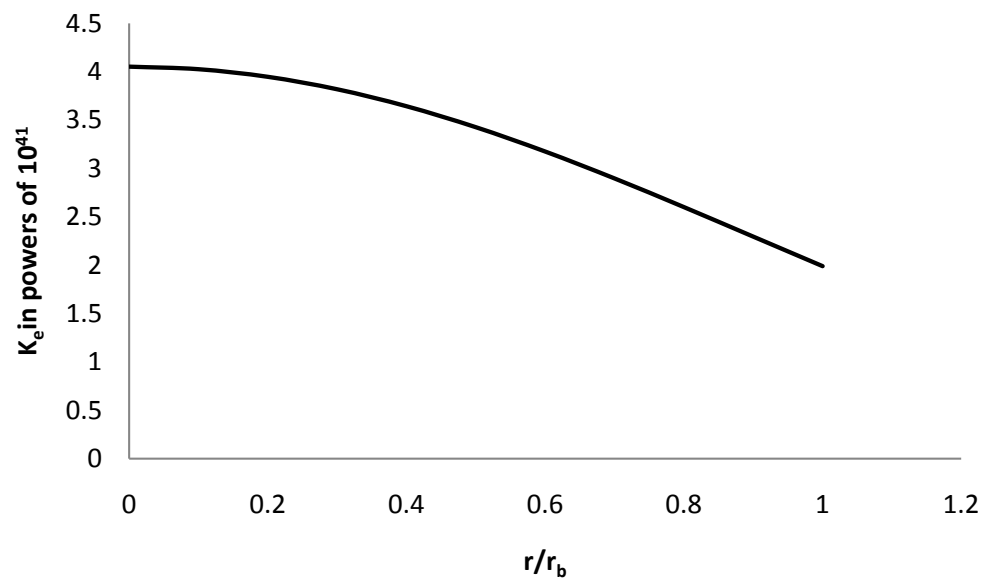

Figure 10. Variation of compression moduli with radius for PSR J0348 + 0432 .

(SEC) is shown in Figure 11. From Figure 12 we observe that adiabatic index is monotonically increasing outward which is necessary for a physically viable 


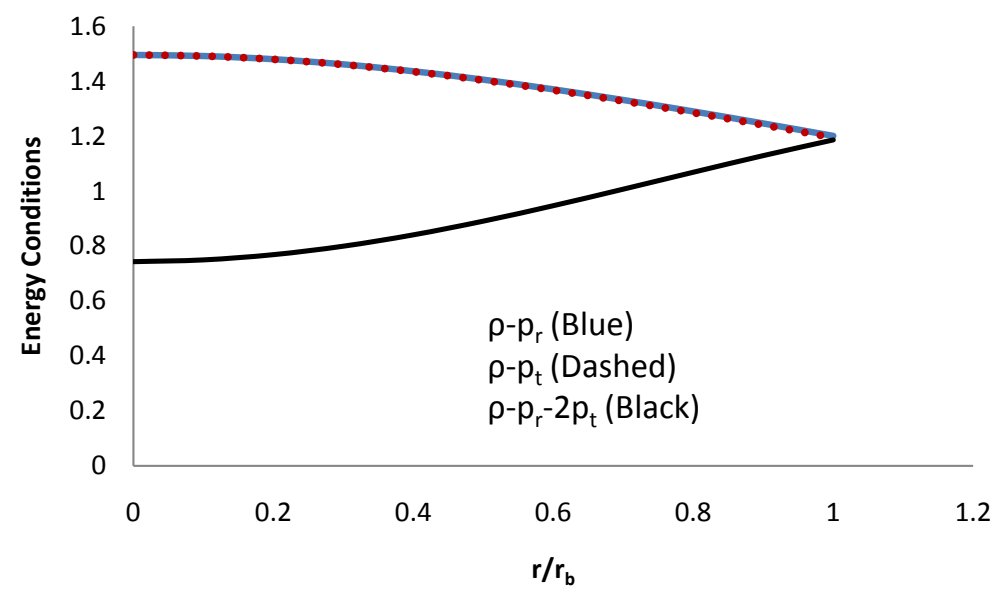

Figure 11. Variation of energy conditions with radius for PSR J0348 +0432 .

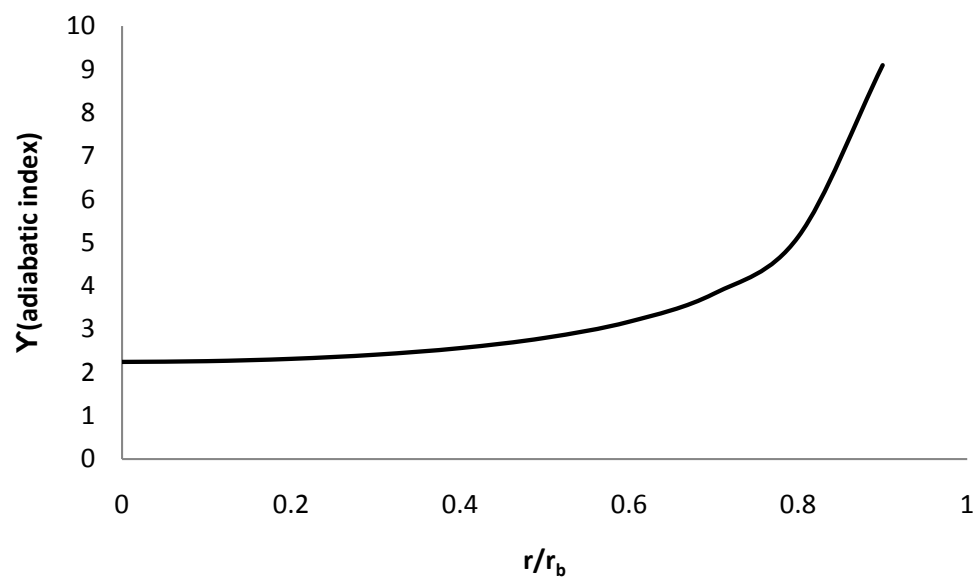

Figure 12. Variation of adiabatic index with radial coordinate for PSR J0348 +0432 .

configuration.

\section{Properties of the New Solution}

The values of pressure and density at centre are given by

$$
\begin{gathered}
{\left[\frac{8 \pi G}{c_{1} c^{4}} p_{r}\right]_{r=0}=\left[\frac{8 \pi G}{c_{1} c^{4}} p_{\perp}\right]=\frac{475}{112}-\frac{\alpha}{2(l-1)}-\frac{k}{m-1}+A>0} \\
{\left[\frac{8 \pi G}{c_{1} c^{2}} \rho\right]=\frac{1935}{112}-\frac{3 k}{1-m}-3 A}
\end{gathered}
$$

At the centre of a star the proper charge density must have a finite value

$$
\sigma(r=0)=\frac{3 c_{1} \sqrt{k}}{4 \pi}>0
$$

The pressure and density also decrease from the centre to the surface

$$
\left[\frac{8 \pi G}{c_{1} c^{4}} \frac{\mathrm{d}^{2} p_{r}}{\mathrm{~d} x^{2}}\right]_{r=0}<0 ;\left[\frac{8 \pi G}{c_{1} c^{4}} \frac{\mathrm{d}^{2} p_{\perp}}{\mathrm{d} x^{2}}\right]_{r=0}<0 ;\left[\frac{8 \pi G}{c_{1} c^{2}} \frac{\mathrm{d}^{2} \rho}{\mathrm{d} x^{2}}\right]_{r=0}<0
$$


The square of speed of sound is given by

$$
v_{r}^{2}=\frac{\mathrm{d} p_{r}}{\mathrm{~d} \rho} ; v_{\perp}^{2}=\frac{\mathrm{d} p_{\perp}}{\mathrm{d} \rho}
$$

$v_{r}^{2} / c^{2}$ and $v_{\perp}^{2} / c^{2}$ must have values less than 1 to fulfill the causality condition and the stability factor must satisfy $-1 \leq v_{\perp}^{2}-v_{r}^{2} \leq 0$. The expression for gravitational red shift and adiabatic index are

$$
z=e^{-v / 2}-1=\frac{(1+x)^{-5 / 2}}{\sqrt{B}}-1 ; \gamma=\left(\frac{p_{r}+\rho}{p_{r}}\right) \frac{\mathrm{d} p_{r}}{\mathrm{~d} \rho}
$$

The gravitational red shift must be non zero and must have positive finite value at the centre. The compression moduli $K_{e}$ is given by

$$
K_{e}=\gamma p_{r}
$$

we can judge the density of compact star with the help of compression moduli, it must have highest value at the centre and should decrease on moving outwards from the centre.

\section{Boundary Conditions}

The interior solution obtained for super dense star must be matched with Reissner-Nordstrom metric at the boundary, the metric is given by

$$
\begin{aligned}
\mathrm{d} s^{2}= & \left(1-\frac{2 G M}{c^{2} r}+\frac{e^{2}}{r^{2}}\right) c^{2} \mathrm{~d} t^{2}-\left(1-\frac{2 G M}{c^{2} r}+\frac{e^{2}}{r^{2}}\right)^{-1} \mathrm{~d} r^{2} \\
& -r^{2}\left(\mathrm{~d} \theta^{2}+\sin ^{2} \theta \mathrm{d} \phi^{2}\right)
\end{aligned}
$$

Here $\mathrm{M}$ represents the mass and e the total charge of compact fluid sphere.

Continuity of $e^{\lambda}, e^{v}$ and $q$ across the boundary $r=r_{b}$ gives the following equations

$$
\begin{gathered}
e^{v_{b}}=\left(1-\frac{2 G M}{c^{2} r_{b}}+\frac{e^{2}}{r_{b}^{2}}\right)=B(1+X)^{5} \\
e^{-\lambda_{b}}=\left(1-\frac{2 G M}{c^{2} r_{b}}+\frac{e^{2}}{r_{b}^{2}}\right)=Y(x)
\end{gathered}
$$

where $X=c_{1} r_{b}^{2}$ and $q=e$ is the charge at $r=r_{b}$. Also the pressure at the boundary $r=r_{b}$

$$
p_{r}\left(r=r_{b}\right)=0
$$

In view of Equation (6.4) the constant A can be computed as

$$
\begin{aligned}
A= & \frac{(1+6 X)^{1 / 3}}{11 X+1}\left(\frac{200 X^{3}+1050 X^{2}+4125 X-475}{112}\right. \\
& \left.-\frac{\alpha(11 X+1)(1+6 X)^{-l / 3}}{2(1-l)}-\frac{k(12 X-m X+1)(1+6 X)^{-m / 3}}{1-m}\right)
\end{aligned}
$$

In view of Equations (6.2) and (6.3) the other constants can be evaluated as 


$$
\begin{aligned}
B= & \frac{1}{(1+X)^{8}}\left(\frac{-8 X^{3}-54 X^{2}-309 X+112}{112}+\frac{\alpha X(1+6 X)^{-l / 3}}{2(1-l)}\right. \\
& \left.+\frac{k X(1+6 X)^{-m / 3}}{1-m}+\frac{A X}{(1+X)^{3}(1+6 X)^{1 / 3}}\right)
\end{aligned}
$$

Mass of super dense star can be expressed as

$$
M=\frac{c^{2} r_{b}}{2 G}\left(1-B(1+X)^{5}+\frac{k X^{2}(1+6 X)^{-m / 3}}{(1+X)^{4}}\right)
$$

The surface density is given by

$$
\begin{aligned}
\frac{8 \pi G}{X c^{2}} \rho_{b} r_{b}^{2}= & \frac{1}{(1+X)^{4}}\left\{\frac{120 X^{3}+450 X^{2}+15 X+1935}{112}\right. \\
& -\frac{k(1+6 X)^{-m / 3-1}}{1-m}\left(-4 m X^{2}-18 X^{2}-4 m X+15 X+3\right) \\
& -\frac{\alpha(1+6 X)^{-l / 3-1}}{2(1-l)}\left(-4 l X^{2}-18 X^{2}-4 l X+15 X+3\right) \\
& \left.-\frac{k X}{(1+6 X)^{m / 3}}-\frac{A}{(1+6 X)^{4 / 3}}\left(-22 X^{2}+11 X+3\right)\right\}
\end{aligned}
$$

In Table 1 we have shown the variation of radial and transverse pressures, density, pressure to density ratio, electric field intensity with respect to radial coordinate $r$ and we observe that these parameters show well behaved nature. In Table 2 the profile of proper charge density, red shift, compression moduli, square of the speed of sound, stability factor and anisotropy have been shown. We see that all these variables have physically reasonable behavior. In Table 3 we have mentioned the well behaved values of the parameters used for two compact stars PSR J0348 + 0432 and EXO 0748-676.

Table 1. The variation of radial and transverse pressures, density, pressure to density ratio and electric field intensity with radial coordinate corresponding to $\mathrm{k}=2.8, \mathrm{X}=0.1, \mathrm{l}=0.1, \mathrm{~m}=0.3, \alpha=1.6$.

\begin{tabular}{cccccccc}
\hline $\mathrm{r} / \mathrm{r}_{\mathrm{b}}$ & $8 \pi \mathrm{G} / \mathrm{c}^{4} \mathrm{p}_{\mathrm{r}} \mathrm{r}_{\mathrm{b}}{ }^{2}$ & $8 \pi \mathrm{G} / \mathrm{c}^{4} \mathrm{p}_{\mathrm{t}} \mathrm{r}_{\mathrm{b}}{ }^{2}$ & $8 \pi \mathrm{G} / \mathrm{c}^{2} \rho \mathrm{r}_{\mathrm{b}}{ }^{2}$ & $\mathrm{p}_{\mathrm{r}} / \rho \mathrm{c}^{2}$ & & $\mathrm{P}_{\mathrm{t}} / \rho \mathrm{c}^{2}$ & $\mathrm{E}^{2} / \mathrm{c}_{1}$ \\
\hline 0 & 0.37607 & 0.37607 & 1.87177 & 0.20091 & 0.20091 & 0 \\
0.1 & 0.37078 & 0.37088 & 1.86255 & 0.19907 & 0.19912 & 0.00099 \\
0.2 & 0.35515 & 0.35554 & 1.83533 & 0.19350 & 0.193723 & 0.00392 \\
0.3 & 0.32987 & 0.33074 & 1.79131 & 0.18415 & 0.18463 & 0.00863 \\
0.4 & 0.29603 & 0.29752 & 1.73233 & 0.17088 & 0.17175 & 0.01487 \\
0.5 & 0.25503 & 0.25728 & 1.66070 & 0.15356 & 0.15492 & 0.02233 \\
0.6 & 0.20848 & 0.21158 & 1.57899 & 0.13203 & 0.13400 & 0.03064 \\
0.7 & 0.15810 & 0.16211 & 1.48982 & 0.10612 & 0.10881 & 0.03943 \\
0.8 & 0.10559 & 0.11053 & 1.39572 & 0.07565 & 0.07919 & 0.04833 \\
0.9 & 0.05258 & 0.05844 & 1.29901 & 0.04048 & 0.04498 & 0.05701 \\
1 & 0.00054 & 0.00726 & 1.20176 & 0.00045 & 0.00604 & 0.06516 \\
\hline
\end{tabular}


Table 2. Variation of proper charge density, red shift, compression moduli, square of the speed of sound, stability factor and anisotropy with radial coordinate with values of $\mathrm{k}=2.8, \mathrm{X}=0.1,=0.1, \mathrm{~m}=0.3, \alpha=1.6$.

\begin{tabular}{ccccccccc}
\hline $\mathrm{r} / \mathrm{r}_{\mathrm{b}}$ & $\sigma$ & $\mathrm{z}$ & $\mathrm{K}_{\mathrm{e}}=\gamma \mathrm{p}_{\mathrm{r}}$ in powers of $10^{41}$ & $\mathrm{v}_{\mathrm{r}}{ }^{2} / \mathrm{c}^{2}$ & $\mathrm{v}_{\mathrm{t}}{ }^{2} / \mathrm{c}^{2}$ & $\mathrm{v}_{\mathrm{t}}{ }^{2} / \mathrm{c}^{2}-\mathrm{v}_{\mathrm{r}}{ }^{2} / \mathrm{c}^{2}$ & $\Delta \mathrm{r}_{\mathrm{b}}^{2}$ \\
\hline 0 & 0.000236 & 0.58550 & 4.05333 & 0.37566 & 0.30491 & -0.070752 & 0 \\
0.1 & 0.000233 & 0.58404 & 4.02687 & 0.37563 & 0.30415 & -0.071481 & 0.0000099 \\
0.2 & 0.000226 & 0.57969 & 3.94817 & 0.37550 & 0.30181 & -0.073682 & 0.000039 \\
0.3 & 0.000215 & 0.57253 & 3.81931 & 0.37511 & 0.29772 & -0.077392 & 0.000086 \\
0.4 & 0.000200 & 0.56272 & 3.6439 & 0.37426 & 0.29159 & -0.082665 & 0.000149 \\
0.5 & 0.000183 & 0.55045 & 3.42711 & 0.37269 & 0.28312 & -0.089568 & 0.000225 \\
0.6 & 0.000164 & 0.53596 & 3.17564 & 0.37012 & 0.27196 & -0.098157 & 0.000310 \\
0.7 & 0.000144 & 0.51951 & 2.89744 & 0.36629 & 0.25783 & -0.108458 & 0.000401 \\
0.8 & 0.000124 & 0.50139 & 2.60127 & 0.36097 & 0.24054 & -0.120424 & 0.000493 \\
0.9 & 0.000104 & 0.48191 & 2.29617 & 0.35392 & 0.22004 & -0.133879 & 0.000585 \\
1 & 0.000085 & 0.46137 & 1.99092 & 0.34498 & 0.19655 & -0.148431 & 0.000672 \\
\hline
\end{tabular}

Table 3. The well behaved values of the parameters used for two compact stars.

\begin{tabular}{cccccccccc}
\hline Compact objects & \multirow{2}{*}{$\alpha$} & $\mathrm{k}$ & $\mathrm{A}$ & $\mathrm{B}$ & $\begin{array}{c}\text { computed value of } \\
\mathrm{R} \mathrm{kms})\end{array}$ & $\begin{array}{c}\text { computed value of } \\
\mathrm{M} / \mathrm{M}_{\Theta}\end{array}$ & \multicolumn{2}{c}{ observed value of } \\
$\mathrm{R}(\mathrm{kms})$ & \multicolumn{2}{c}{$\begin{array}{c}\text { observed value of } \\
\mathrm{M} / \mathrm{M}_{\Theta}\end{array}$} \\
\hline PSR J0348 + 0432 & \multirow{2}{*}{1.6} & 2.8 & -1.96 & 0.49 & 14.51 & 2.17 & $13 \pm 2$ & 2.01 \\
EXO 0748 - 676 & 1.8 & 3.2 & -1.96 & 0.52 & 13.73 & 1.62 & $13.8 \pm 1.8$ & $2.1 \pm 0.3$ \\
\hline
\end{tabular}

\section{Results and Discussions}

In the present article we have proposed a new model of anisotropic charged compact star which satisfies all the physical reality conditions and is free from central singularity with the exterior space time being the Reissner-Nordstrom metric. We electrified the uncharged fluid sphere e.g. Durgapal $V^{\text {th }}$ solution [1] with anisotropy taken into account. The charged solution is well behaved with positive values of charge parameter $k$.

The regularity of metric potentials throughout the interior of the star can be observed from Figure 1 by showing that the solution is free from geometrical or physical singularity. It has been observed from the Figures 2-7 that the physical parameters $p_{r}, p_{\perp}, \rho, p_{r}, \rho, p_{\perp}, \rho, z, \sigma$ are positive inside the stellar model and they are monotonically decreasing function of radial coordinate. At the boundary of the star the radial pressure vanishes while the matter density and transverse pressure are non negative. The electric field intensity and adiabatic index $\gamma$ also increase with radial coordinate by which we conclude that this is a physically viable configuration Figure 4 and Figure 12. Further the adiabatic index is more than $4 / 3$ throughout the interior of the star, which reconfirms the stability of our model. We also observe that the anisotropic factor $\Delta>0$ and increases when we move from centre towards boundary as observed from Figure 9. Moreover at the centre of a star $\Delta$ vanishes which is an essential feature of a realistic star. The profile of velocity of sound has been shown in Figure 8. For 
our present model both radial and transverse velocity are less than 1 and monotonically decreasing towards the boundary which shows that causality condition holds good inside the star. From Figure 10 it is also observed that the compression moduli of star decreases with the increase of radius which indicates that star is more dense at the centre than outer surface. Furthermore our new model for anisotropic fluid distribution also satisfies the null energy condition $(\rho>0)$, weak energy condition $\left(\rho-p_{r}\right) \geq 0$ and $\left(\rho-p_{t}\right) \geq 0$ and the strong energy condition $\rho-p_{r}-2 p_{t} \geq 0$ Figure 11. We have shown the variation of required physical parameters with radial coordinate corresponding to $k=2.8, X=0.1$, $l=0.1, \quad m=0.3, \quad \alpha=1.6$ in Table 1 and Table 2 . Finally we have constructed a neutron star model by assuming surface density $2 \times 10^{14} \mathrm{gm} / \mathrm{cm}^{3}$ corresponding to, $\alpha=1.6, X=0.1$ and $k=2.8$ with mass $2.17 M_{\Theta}$ and radius $14.51 \mathrm{kms}$ which is close to the observational data of the neutron star PSR J0348 + 0432 [53]. The parameters with values $\alpha=1.8, X=0.1$ and $k=3.2$ generates the model for EXO 0748-676 with radius $13.73 \mathrm{kms}$ and mass $1.62 M_{\Theta}$ [54]. We have provided the well behaved parameters of these two well known compact stars which gives mass and radius compatible to observed values in $\mathrm{Ta}$ ble 3 .

\section{References}

[1] Durgapal, M.C. (1982) Journal of Physics A: Mathematical and General, 15, 2637. https://doi.org/10.1088/0305-4470/15/8/039

[2] Witten, E. (1984) Physical Review D, 30, 272. https://doi.org/10.1103/PhysRevD.30.272

[3] Bodmer, A. (1971) Physical Review D, 4, 1601. https://doi.org/10.1103/PhysRevD.4.1601

[4] Schwarzschild, K. (1916) On the Gravitational Field of a Mass Point According to Einstein's Theory. Sitzbcr. Press. Akad. Wiss. Berlin, 189.

[5] Schwarzschild, K. (1916) Uber das Gravitationsfeld einer kugel aus inkompressibler Flussigkeit nach der Einsteinsachen Theorie. Sitzbcr. Press. Akad. Wiss. Berlin, 424.

[6] Ivanov, B.V. (2002) Physical Review D, 65, 104001. https://doi.org/10.1103/PhysRevD.65.104001

[7] Bonner, W.B. (1965) Monthly Notices of the Royal Astronomical Society, 137, 239. https://doi.org/10.1093/mnras/129.6.443

[8] Pant, N. and Rajasekhara, S. (2011) Astrophysics Space Science, 333, 161-168. https://doi.org/10.1007/s10509-011-0607-z

[9] Kiess, T.E. (2012) Astrophysics Space Science, 339, 329-338. https://doi.org/10.1007/s10509-012-1013-x

[10] Murad, M.H. and Fatema, S. (2015) The European Physical Journal Plus, 130, 3.

[11] Canuto, V. and Chitre, M. (1973) Physical Review Letters, 30, 999-1002.

[12] Canuto, V. and Chitre, M. (1974) Physical Review D, 9, 1587-1613.

[13] Canuto, V. (1974) Annual Review of Astronomy and Astrophysics, 12, 167-214.

[14] Canuto, V. (1975) Annual Review of Astronomy and Astrophysics, 13, 335-380.

[15] Weber, F. (1999) Pulsars as Astrophysical Observatories for Nuclear and Particle 
Physics. Institute of Physics Publishing, Bristol.

[16] Wolf, R.A. (1966) The Astrophysical Journal, 145, 834. https://doi.org/10.1086/148829

[17] Ruderman, M. (1972) Annual Review of Astronomy and Astrophysics, 10, 427. https://doi.org/10.1146/annurev.aa.10.090172.002235

[18] Sawyer, R.F. (1972) Physical Review Letters, 29, 382. https://doi.org/10.1103/PhysRevLett.29.382

[19] Sokolov, A.I. (1980) JETP Letters, 79, 1137.

[20] Dev, K. and Gleiser, M. (2003) General Relativity and Gravitation, 35, 1435. https://doi.org/10.1023/A:1024534702166

[21] Bowers, R.L. and Liang E.P.T. (1974) The Astrophysical Journal, 188, 657-665.

[22] Bayin, S.S. (1982) Physical Review D, 26, 1262-1274.

[23] Krori, K.D., Borgohaiann, P. and Devi, R. (1984) Canadian Journal of Physics, 62, 239-246.

[24] Herrera, L. and Ponce de Leon, J. (1985) Journal of Mathematical Physics, 26, 2302-2307.

[25] Ponce de Leon, J. (1987) Journal of Mathematical Physics, 28, 1114. https://doi.org/10.1063/1.527557

[26] Ponce de Leon, J. (1987) General Relativity and Gravitation, 19, 797. https://doi.org/10.1007/BF00768215

[27] Bondi, H. (1992) Monthly Notices of the Royal Astronomical Society, 259, 365-368.

[28] Gokhroo, M.K. and Mehra, A.L. (1994) General Relativity and Gravitation, 26, 75. https://doi.org/10.1007/BF02088210

[29] Patel, L.K. and Mehta, N.P. (1995) Australian Journal of Physics, 48, 635-643.

[30] Bhar P. and Ratanpal, B.S. (2016) Astrophysics and Space Science, 361, 217

[31] Herrera, L., Prisco, A.D., Ospino, J. and Fuenmayor, E. (2001) Journal of Mathematics, 42, 2129-2143. https://doi.org/10.1063/1.1364503

[32] Chaisi, M. and Maharaj, S.D. (2006) Pramana Journal of Physics, 66, 313-324.

[33] Bhar, P., et al. (2016) Astrophysics and Space Science, 361, 284.

[34] Karmakar, S., Mukherjee, S., Sharma, R. and Maharaj, S.D. (2007) Pramana Journal of Physics, 68, 881-889.

[35] Sharma, R. and Maharaj, S.D. (2007) Monthly Notices of the Royal Astronomical Society, 375, 1265-1268.

[36] Herrera, L. and Santos, N. (1997) Physics Reports, 286, 53-130.

[37] Thirukkanesh, S. and Ragel, F.C. (2014) Astrophysics and Space Science, 354, 415. https://doi.org/10.1007/s10509-014-1883-1

[38] Maurya, S.K. and Gupta, Y.K. (2014) Astrophysics and Space Science, 353, 657. https://doi.org/10.1007/s10509-014-2041-5

[39] Pandya, D.M., Thomas, V.O. and Sharma, R. (2015) Astrophysics and Space Science, 356, 285. https://doi.org/10.1007/s10509-014-2207-1

[40] Bahar, P., Murad, M.H. and Pant, N. (2015) Astrophysics and Space Science, 359, 13. https://doi.org/10.1007/s10509-015-2462-9

[41] Murad, M.H. (2016) Astrophysics and Space Science, 361, 20. https://doi.org/10.1007/s10509-015-2582-2

[42] Herrera, L., Prisco, A.D., Ospino, J. and Fuenmayor, E. (2001) Journal of Mathe- 
matical Physics, 42, 2129. https://doi.org/10.1063/1.1364503

[43] Herrera, L., Ospino, J. and Prisco, A.D. (2008) Physical Review D, 77, Article ID: 027502. https://doi.org/10.1103/PhysRevD.77.027502

[44] Chaisi, M. and Maharaj, S.D. (2006) Pramana Journal of Physics, 66, 609-614.

[45] Maurya, S.K. and Gupta, Y.K. (2012) Physica Scripta, 86, Article ID: 025009. https://doi.org/10.1088/0031-8949/86/02/025009

[46] Maurya, S.K. and Gupta, Y.K. (2013) Astrophysics and Space Science, 344, 243. https://doi.org/10.1007/s10509-012-1302-4

[47] Maharaj, S.D. and Govender, M. (2005) International Journal of Modern Physics D, 14, 667. https://doi.org/10.1142/S0218271805006584

[48] Pant, N., et al. (2014) Astrophysics and Space Science, 352, 135-141. https://doi.org/10.1007/s10509-014-1904-0

[49] Pradhan, N. and Pant, N. (2015) Astrophysics and Space Science, 356, 67. https://doi.org/10.1007/s10509-014-2198-y

[50] Singh, K.N., et al. (2014) International Journal of Theoretical Physics, 54, 3408.

[51] Pant, et al. (2015) Astrophysics and Space Science, 355, 137-145. https://doi.org/10.1007/s10509-014-2156-8

[52] Maharaj, S.D., Sunzu, J.M. and Ray, S. (2014) The European Physical Journal Plus, 129. https://doi.org/10.1140/epjp/i2014-14003-9

[53] Demorest, P.B., et al. (2010) Nature, 467, 1081. https://doi.org/10.1038/nature09466

[54] Mak, M.K. and Harko Chin, T. (2002) Astronomy \& Astrophysics, 2, 248. 